\title{
Synthesis and Characterisation of ETS-10/Acetate-based Ionic Liquid/Chitosan Mixed Matrix Membranes for $\mathrm{CO}_{2} / \mathrm{N}_{2}$ Permeation
}

\section{Clara Casado-Coterillo ${ }^{1, *}$, María del Mar López-Guerrero ${ }^{2}$ and Ángel Irabien ${ }^{1}$}

1 Department of Chemical and Biomolecular Engineering, Universidad de Cantabria, Avda. Los Castros s/n, 39005 Santander, Spain; E-Mail: irabienj@unican.es

2 Department of Analytical Chemistry, Faculty of Sciences, Universidad de Málaga, 29071 Málaga, Spain; E-Mail: mmlopez@uma.es

* Author to whom correspondence should be addressed; E-Mail: casadoc@unican.es; Tel.: +34-942-206777.

Received: 2 April 2014; in revised form: 19 May 2014 / Accepted: 12 June 2014 /

Published: 19 June 2014

\begin{abstract}
Mixed matrix membranes (MMMs) were prepared by incorporating organic surfactant-free hydrothermally synthesised ETS-10 and 1-ethyl-3-methylimidazolium acetate ionic liquid (IL) to chitosan (CS) polymer matrix. The membrane material characteristics and permselectivity performance of the two-component membranes were compared with the three-component membrane and the pure CS membrane. The addition of IL increased $\mathrm{CO}_{2}$ solubility of the polymer, and, thus, the $\mathrm{CO}_{2}$ affinity was maintained for the MMMs, which can be correlated with the crystallinity, measured by FT-IR, and void fraction calculations from differences between theoretical and experimental densities. The mechanical resistance was enhanced by the ETS-10 nanoparticles, and flexibility decreased in the two-component ETS-10/CS MMMs, but the flexibility imparted by the IL remained in three-component ETS-10/IL/CS MMMs. The results of this work provide insight into another way of facing the adhesion challenge in $\mathrm{MMMs}$ and obtain $\mathrm{CO}_{2}$ selective MMMs from renewable or green chemistry materials.
\end{abstract}

Keywords: mixed matrix membranes; chitosan; microporous titanosilicate ETS-10; 1-ethyl-3-methyl-imidazolium acetate ionic liquid; membrane characterisation 


\section{Introduction}

The separation and capture of $\mathrm{CO}_{2}$ from flue gas is becoming important for greenhouse emission control and strong demand of more energy-, cost-effective and environmentally friendly technologies are growing. Membrane-based gas separation has been postulated to compete with absorption in terms of energy requirement when $\mathrm{CO}_{2}$ content in the feed is larger than $20 \%$, as in cement or steel factories [1], due to its low energy consumption, easy operation, and low maintenance.

The membrane is the key element since the separation phenomenon occurs in it, thus, the material used in the membrane preparation determines the separation performance. Depending on the material, membranes are usually classified as polymeric, inorganic, and, more recently, mixed matrix membranes (MMMs). Transport through a dense-polymeric membrane usually takes place through the solution-diffusion mechanism in three steps: (i) the selective component adsorbs in the membrane; (ii) where diffuses through; and (iii) the component desorbs from the other side, due to the low pressure kept at the permeate side. Commercial polymer membranes are relatively easily processed at low costs, but their limited resistance to high temperature, usual inadequacy to high flow rates, or sensitiveness to clogging by dust, there is an absence of economy of scale and low selectivity to $\mathrm{CO}_{2} / \mathrm{N}_{2}$ separation [2]. Inorganic membranes have good catalytic and separation behaviour and present good chemical and temperature resistance. The transport mechanism depends usually on the pore size distribution of the selective layer and, although there are several inorganic membranes commercially available for pervaporation and vapour permeation and liquid filtration processes, not yet for gas separation [3]. The reproducibility and fabrication cost is still a major challenge [4].

Morphology issues have been addressed in many different membrane materials, from pure zeolite membranes [5], to hollow fibre [6], or composite membranes [7]. MMMs are heterogeneous materials formed by the combination of an organic polymer continuous matrix and inorganic material dispersed phase, with the aim of obtaining a well-dispersed heterogeneous mixture of synergistic properties and overcoming the accepted trade-off between permeability and selectivity for gas separation membranes [8,9]. Permeability is a unit flux defining the gas molecules a membrane lets go through, normalized by operation factors such as pressure and thickness. Thus, permeation takes place because of a chemical potential difference across the membrane, which means that the driving force is usually partial pressure difference. To simplify the understanding of the system, the permeability is calculated as the product of solubility $(S)$ and diffusivity $(D)$. The solubility coefficient is given by the condensability of the penetrant, i.e., $\mathrm{CO}_{2}$ in the case of the present work, in membrane material, and the diffusivity coefficient depends on the size and shape of $\mathrm{CO}_{2}$, the free volume or porosity and pore size distribution and the chain flexibility of the polymer. Adhesion between dispersed and continuous phases is the main challenge in MMMs, leading to several morphologies as those represented in Figure 1 , thus affecting separation performance. Figure $1 \mathrm{a}$ is the schematic representation of the ideal morphological contact among inorganic fillers and polymer. Figure $1 \mathrm{~b}$ refers to the non-ideal detachment between inorganic fillers and polymer matrix, creating voids around the former that alter the gas perm-selectivity performance. Figure 1c corresponds to the non-ideal free volume reduction by polymer chain rigidification and pore blockage. In order to improve adhesion, several strategies have been studied, such as functionalization of the inorganic particles prior to introduction into the polymer matrix, or the incorporation of an ionic liquid in the membrane matrix [10,11]. The novelty of this 
work consists in attempting a similar approach but using a non-toxic ionic liquid, a biopolymer, and a microporous zeo-type material prepared without costly organic surfactant.

Figure 1. Schemes of the most typical nanoscale morphologies in MMM structures adapted for ETS-10 in the chitosan polymer membrane: ideal morphology (a); "sieve-in-a-cage" presence of interfacial voids (b); and rigidification by free volume reduction (c).



(a)

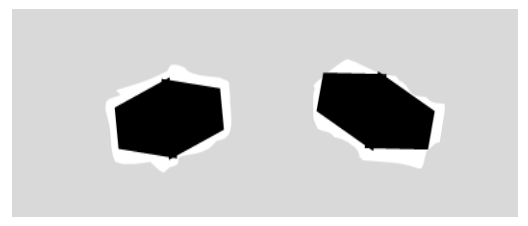

(b)

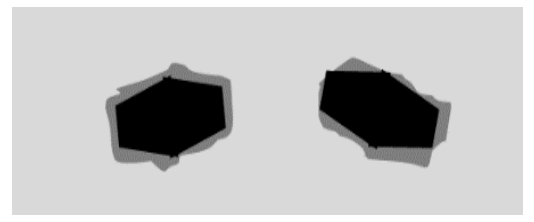

(c)

The continuous polymer chosen is chitosan $(\mathrm{CS})$, poly[ $\beta(1 \rightarrow 4)$-2-amino-2-deoxy-D-glucopyranose], a linear polysaccharide obtained by the deacetylation of chitin, an abundant natural polymer, cheap and obtained from renewable sources, i.e., the shell of crustaceans. CS is biodegradable, biocompatible, non-toxic, and hydrophilic. The high hydrophilicity of chitosan makes it prone to hydrate and form water-swollen membranes with enhanced $\mathrm{CO}_{2}: \mathrm{N}_{2}$ perm-selectivity because of the high $\mathrm{CO}_{2}$ solubility in water [12]. Swollen CS-based membranes have been studied for $\mathrm{CO}_{2}$ separation, usually by humidifying the feed gas prior to entering the membrane module [13] for $\mathrm{CO}_{2}: \mathrm{N}_{2}$ 50:50 (vol \%) mixtures and recently the influence of free and bound water on separation performance was analysed, for diluted $\mathrm{CO}_{2}: \mathrm{N}_{2}: \mathrm{H}_{2}$ mixtures simulating flue gas streams [14,15]. Its mechanical stability has, nevertheless, been tried to improve by coating on a porous polysulfone support [16], organic chemical crosslinking [17], and physical mixing with zeolite particles [18]. Facilitated transport in the solid matrix is expected to increase the stability as well, and CS, because of the weak acid-base interactions between $\mathrm{CO}_{2}$ and water molecules and the amino groups in the chains, has potential to enhance the electrostatic interactions among permeating molecules and the functional groups in the polymer by introducing appropriate materials.

The structure of the microporous titanosilicate ETS-10 is made of orthogonal $\mathrm{TiO}_{6}$ octahedra and $\mathrm{SiO}_{2}$ tetrahedra linked by oxygen atoms shared in the corners. Ti atoms in a six-coordinated state have two negative charges balanced by $\mathrm{Na}^{+}$and $\mathrm{K}^{+}$[19]. The high cation exchange capacity is what makes ETS-10 very interesting in adsorption [20], catalysis, and membrane separation processes [21]. ETS-10 can be synthesised in different sizes including nano-scale [19], which may be homogeneously dispersed in a polymer providing this with its intrinsic characteristics.

Ionic liquids (ILs) combining good and tuneable solubility properties with negligible vapour pressure and good thermal stability have recently received much attention as green solvents and $\mathrm{CO}_{2}$ absorbents in supported liquid membrane contactors [22]. The $\mathrm{CO}_{2}$ solubility is higher when acetate is the anion and the shorter length of the cation, and 1-ethyl-3-methylimidazolium acetate, [emim][Ac], the room temperature ionic liquid (IL) with the highest reported $\mathrm{CO}_{2}$ solubility [23], as well as non-reported toxicity [24], was chosen for the proof-of-concept of this work. A good interaction with CS is expected since it has been reported as a good solvent for polysaccharides [25], because of the strong H-bonds forming with the $\mathrm{OH}$ groups in the polymer chain. $\mathrm{CS}$ and chitin have been reported to enhance the $\mathrm{CO}_{2}$ solubility of low absorbing [bmim][Cl] because the ionic liquid is able to alter the 
$\mathrm{H}$-bonds in the polymer chains, thus freeing amino groups that become available for $\mathrm{CO}_{2}$ absorbing sites [26].

Table 1 shows the chemical formula and properties of all the components.

Table 1. Main properties of the mixed matrix membranes (MMM) components used in this work.

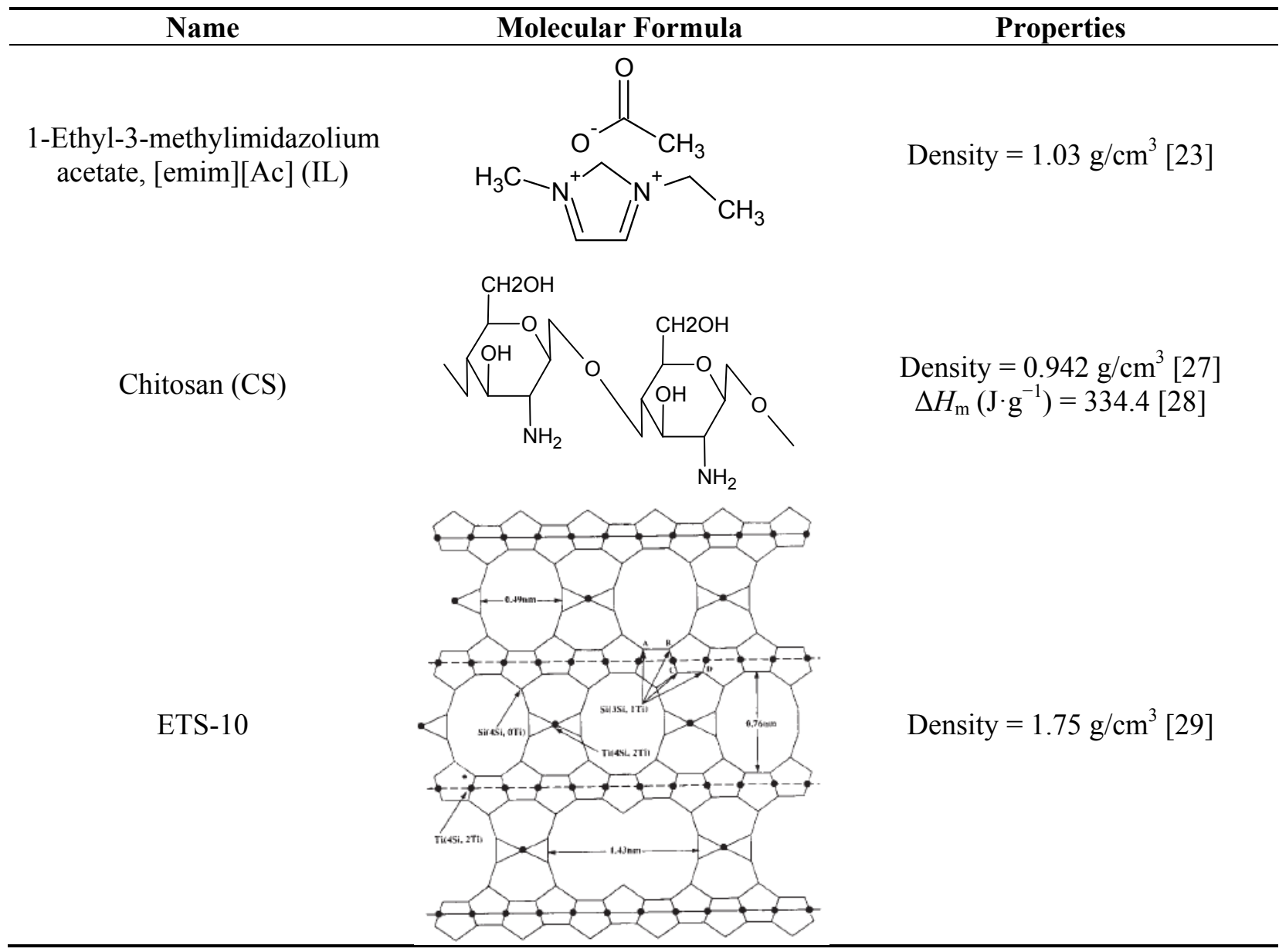

In this work, novel MMMs composed of CS, IL/CS, ETS-10/CS and ETS-10/IL/CS, with the small loading of dispersed filler of $5 \mathrm{wt} \%$ with respect to the continuous polymer matrix, were prepared and tested for $\mathrm{CO}_{2}$ and $\mathrm{N}_{2}$ permeation. Factors affecting membrane morphology such as adhesion, interaction among components, and thermal, chemical, and mechanical resistance have been explored and discussed.

\section{Results and Discussion}

\subsection{Thermal Properties}

The thermal properties of the MMMs are shown in the TGA diagrams in Figure 2. The major weight loss for all the materials involved in membrane preparation occurs at a temperature between 250 and $630{ }^{\circ} \mathrm{C}$. This weight loss is due to the decomposition of the matrix. The thermal decomposition of the IL occurs at $200{ }^{\circ} \mathrm{C}$, which agrees with literature [23]. The decomposition of chitosan powder $\left(560^{\circ} \mathrm{C}\right)$ agrees with literature [30], where the degradation of CS is established in two 
stages and decomposition temperature is considered at the end of the first stage $\left(540{ }^{\circ} \mathrm{C}\right)$. Regarding the CS-based membranes prepared in this work, the pristine CS membrane has almost the same decomposition temperature as the precursor chitosan powder $\left(560{ }^{\circ} \mathrm{C}\right)$, which is increased to more than $620{ }^{\circ} \mathrm{C}$ upon introduction of ETS-10 inorganic particles, even at the low loading of $5 \mathrm{wt} \%$ used in this work. This agrees with the decomposition peak of the second stage that is observed at 564, 579, 593, and $604{ }^{\circ} \mathrm{C}$ for the CS, IL/CS, ETS-10/CS, and ETS-10/IL/CS membranes, respectively, but not observed for the CS powder.

Figure 2. TGA curves of the CS and CS-hybrid membranes. TGA of CS powder, ETS-10 crystalline particles and IL are also included for comparison. DTG curves are presented in the inset.

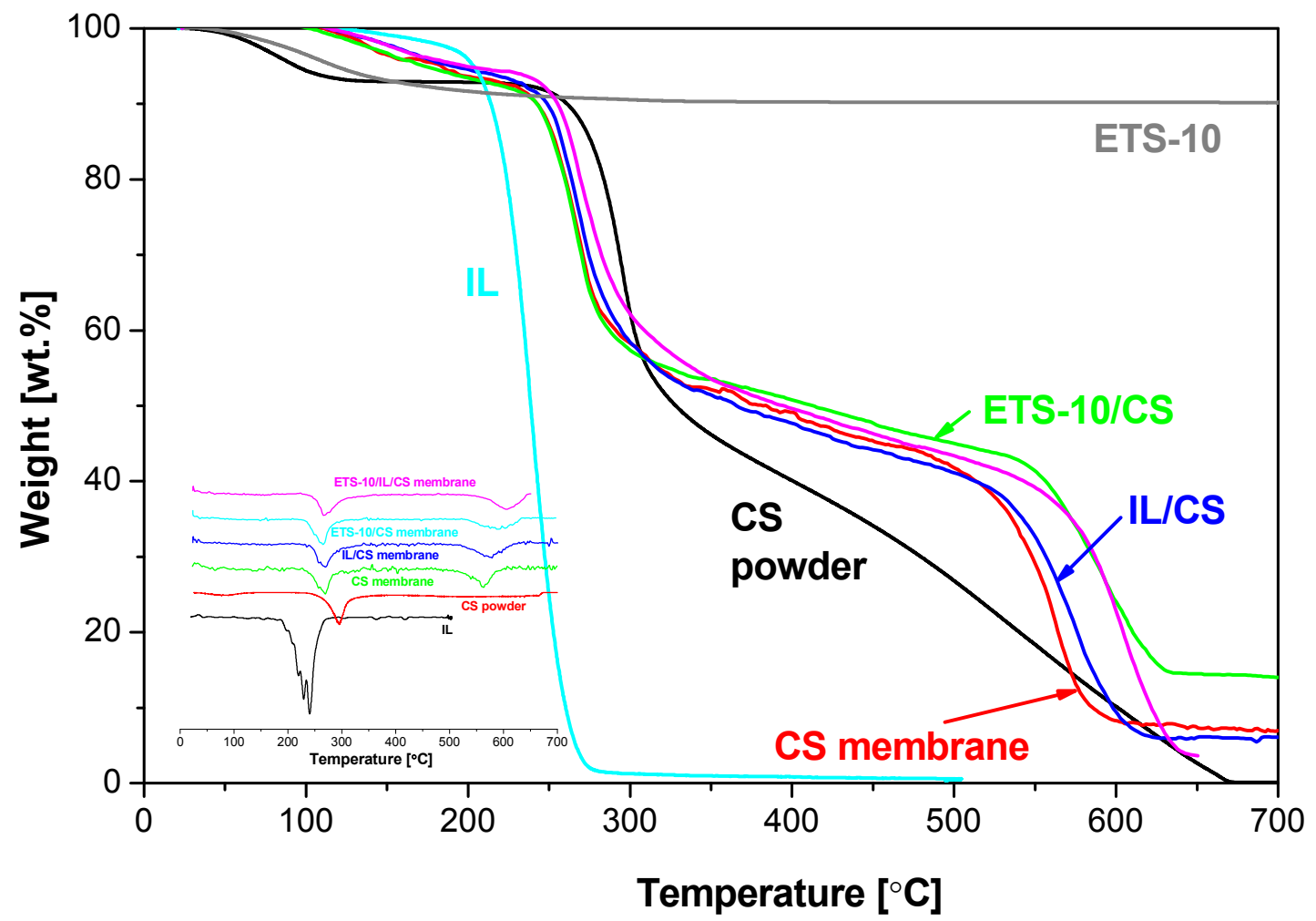

The shifting of the degradation temperature of the CS membranes upon addition of IL and ETS-10 is an indication of enhanced thermal stability, which may be of the potential of these novel membrane materials for higher temperature $\mathrm{CO}_{2}$ separation.

Figure 3 shows the DSC plot of the CS-based membranes prepared in this work, which is the usually reported technique to provide information regarding change from rubbery to glassy state in a polymer material, and this is the method for estimating the crystallinity of semi-crystalline polymers. In Figure 3, only ETS-10/CS MMM allows discerning a glass transition temperature around $196{ }^{\circ} \mathrm{C}$, which agrees with previous observations [18], because the glass transition of CS is generally reported difficult to discern by DSC because of the huge hydrophilicity [17]. 
Figure 3. DSC plots of CS-based membranes.



\subsection{Mechanical Properties}

Table 2 collects the mechanical properties of the CS and MMMs studied in this work. The tensile strength of pure CS membranes agrees with values reported in literature [31], despite differences on the precursor CS powder employed and membrane preparation protocols, which account from the natural origin of this material.

Table 2. Conditions of the tensile tests (General conditions: sample width $5 \mathrm{~mm}$, grip to grip distance $40.20 \pm 8.76 \mathrm{~mm}$ ).

\begin{tabular}{ccccc}
\hline $\begin{array}{c}\text { Membrane } \\
\text { Material } \\
\text { Composition }\end{array}$ & $\begin{array}{c}\text { Thickness } \\
(\boldsymbol{\mu m})\end{array}$ & $\begin{array}{c}\text { Filler Content } \\
\mathbf{( w t ~ \% )}\end{array}$ & $\begin{array}{c}\text { Tensile Strength } \\
\mathbf{( M P a )}\end{array}$ & $\begin{array}{c}\text { Elongation at } \\
\text { Break (\%) }\end{array}$ \\
\hline CS & $121.9 \pm 3.96$ & 0 & $31.63 \pm 7.41$ & $18.52 \pm 8.23$ \\
IL/CS & $128.0 \pm 3.57$ & 5 & $16.09 \pm 11.04$ & $40.44 \pm 12.45$ \\
ETS-10/CS & $130.0 \pm 4.50$ & 5 & $24.30 \pm 4.88$ & $14.40 \pm 9.38$ \\
ETS-10/IL/CS & $168.0 \pm 5.0$ & $5($ each) & $19.93 \pm 5.01$ & $36.15 \pm 3.03$ \\
\hline
\end{tabular}

The tensile strength diminishes upon addition of IL and ETS-10 particles. This was attributed to plasticisation of the polymer matrix reflected by the large increase on the value of the elongation at break, for the IL/CS with respect to CS membranes. The introduction of [bmim] $\left[\mathrm{CF}_{3} \mathrm{SO}_{3}\right]$ IL in semi crystalline Pebax polymers [32] caused a decrease in elongation at break that has not been observed here. On the other hand, the elongation at break of the CS based membranes decreases upon addition of ETS-10 particles. This is due to the rigidification of the organic polymer [33] by the addition of inorganic fillers.

\subsection{Structural and Morphological Properties}

The increased flexibility imparted to the MMMs by the introduction of the IL was attributed to the singular interaction between CS and IL, and that was the reason to measure FT-IR spectra of the membranes (Figure 4). The CS and 3-component ETS-10/IL/CS membranes demonstrated a broad 
band in the range $3600-2700 \mathrm{~cm}^{-1}$, attributed to $\mathrm{NH}$ and $\mathrm{OH}$ vibrations, whereas the broad band at around $2940 \mathrm{~cm}^{-1}$ in CS is shifted to lower wave numbers due to film formation. In the second region, bands attributed to amide I $\left(1633 \mathrm{~cm}^{-1}\right)$, amide II $\left(1537 \mathrm{~cm}^{-1}\right)$ and amide III $\left(1315 \mathrm{~cm}^{-1}\right)$ in CS [34], decreased in intensity, and are mixed up with the microporous titanosilicate ETS-10 and 1-ethyl-3methyl-imdazolium acetate IL own bands upon hybridisation. This confirms the good interaction existing among the components involved on MMM preparation, and that may account for the higher flexibility of the hybrid membrane materials imparted to both CS and ETS-10/CS MMMs, as explained above regarding mechanical properties of the membranes in Table 2.

Figure 4. FT-IR measurements of CS-based membranes.

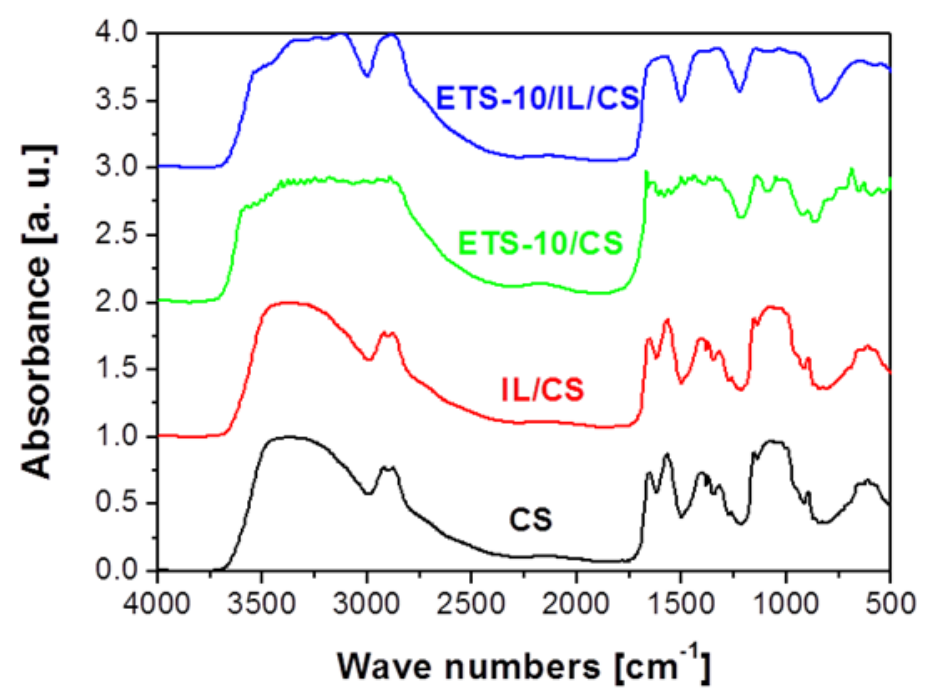

The crystallinity, $\chi$, of the CS-based membrane samples was calculated from the FTIR spectra, using the ratio of the absorbance at 1423 and $890 \mathrm{~cm}^{-1}$, respectively. The values are collected in the last column of Table 3, together with other morphological properties of the membranes. The crystallinity increased monotonously upon addition of IL, ETS-10 and both to the CS polymer matrix, as reported for other MMMs prepared from semi crystalline polymers [35], which attribute this phenomenon to the role of inorganic fillers as nucleating agents. The crystallinity is important in membrane analysis since the amorphous part of the polymer is the main contributor to the gas transport.

Table 3. Morphological properties of CS-based membranes: Theoretical density ( $\left.\rho_{\text {add }}\right)$, measured density $\left(\rho_{\mathrm{m}}\right)$, void volume fraction $\left(\phi_{\mathrm{v}}\right)$ and true dispersed filler volume fraction.

\begin{tabular}{cccccc}
\hline $\begin{array}{c}\text { Membrane } \\
\text { materials }\end{array}$ & $\begin{array}{c}\boldsymbol{\rho}_{\text {add }} \\
\left(\mathbf{g} / \mathbf{c m}^{3}\right)\end{array}$ & $\begin{array}{c}\boldsymbol{\rho}_{\mathbf{m}} \\
\left(\mathbf{g} / \mathbf{c m}^{3}\right)\end{array}$ & $\begin{array}{c}\boldsymbol{\phi}_{\mathbf{v}} \\
(\mathbf{v o l} / \mathbf{v o l})\end{array}$ & $\begin{array}{c}\boldsymbol{\phi}_{\mathbf{d}} \\
(\mathbf{v o l} / \mathbf{v o l})\end{array}$ & $\chi(-)$ \\
\hline CS & 0.942 & $0.727 \pm 0.26$ & 0.228 & 0.520 & 0.14 \\
IL/CS & 0.941 & $1.108 \pm 0.48$ & -0.165 & 0.032 & 0.18 \\
$\mathrm{ETS}-10 / \mathrm{CS}$ & 1.014 & $0.637 \pm 0.14$ & 0.372 & 0.028 & 0.28 \\
$\mathrm{ETS}-10 / \mathrm{IL} / \mathrm{CS}$ & 1.442 & $0.714 \pm 0.14$ & 0.505 & 0.304 & 0.34 \\
\hline
\end{tabular}

The theoretical density $\left(\rho_{\text {add }}\right)$ of the membrane was calculated using the additive model, as follows, 


$$
\rho_{\text {add }}=\frac{1}{\frac{1-w_{d}}{\rho_{c}}+\frac{w_{d}}{\rho_{d}}}
$$

where $w_{\mathrm{d}}$ is the weight fraction of the dispersed phase (IL or ETS-10) in the continuous matrix, $\rho_{\mathrm{d}}$ and $\rho_{c}$ are the densities of the dispersed and the polymer phase in the continuous matrix in Table 1.

The nominal volume fraction of the filler, $\phi_{d}^{N}$, is described as:

$$
\phi_{d}^{N}=\frac{V_{d}}{V_{c}+V_{d}}
$$

where $V_{\mathrm{d}}$ and $V_{\mathrm{c}}$ are the ideal contributions of the dispersed and continuous phases, the inorganic filler and organic continuous matrix, respectively, to the total volume, defined as the mass of the organic and inorganic components added to the composite, divided by the pure polymer or filler density. In this work, the CS polymer or IL/CS hybrid are considered as continuous matrix for the 2- and 3-component MMMs, respectively.

The measured density values are always smaller than the theoretical values. This accounts for the void fraction created in the MMM upon addition of the porous ETS-10 nanoparticles, which resulted in lower density. This is the reason why the ideal MMM morphology, where a perfect adhesion between the organic and the inorganic phase, as depicted in Figure 1a, is seldom present in reality and the non-ideal free-volume in Figure 1b, attributed to poor interfacial contact between dispersed and continuous phases (Figure 1c), must be taken into account. The true filler volume fraction, $\phi_{\mathrm{d}}$, was calculated by comparing the experimental and the theoretical densities, when the void volume fraction can be calculated as [35]:

$$
\begin{gathered}
\phi_{d}=\phi_{d}^{N}\left(1-\phi_{v}\right) \\
\phi_{v}=1-\frac{\rho_{m}}{\rho_{a d d}}
\end{gathered}
$$

The void fraction increased significantly for the two-component ETS-10/CS and the three-component ETS-10/IL/CS MMMs above that of CS membranes. This increase can be attributed to several factors: polymer crystallinity, interphase voids between the particles and the porosity of the crystals themselves $[35,36]$. The highest value of the density for the IL/CS membrane and the negative value for the void volume fraction may be attributed to the intimate contact between IL and CS.

Table 4 summarises the solubility values calculated from thermo gravimetrical sorption experiments. It is worth noticing that the solubility values of the single components do not agree with the additive rule, which correlates with the observation of non-idealities and void fraction calculations in Table 3, in agreement with the MMM morphologies in Figure 1. The $\mathrm{CO}_{2}$ solubility increases upon addition of ETS-10 particles, which agrees with our assumption that the incorporation of this alkaline titanosilicate favours $\mathrm{CO}_{2}$ permeation due to its adsorption affinity toward $\mathrm{CO}_{2}$. The $\mathrm{N}_{2}$ solubility decreased about $42 \%$ in ETS-10/CS MMMs, which was attributed to the molecular sieve effect of the nanoporous titanosilicate. The average pore opening of ETS-10 is $0.8 \mathrm{~nm}$ [37]. The $\mathrm{N}_{2}$ solubility in the three-component ETS-10/IL/CS MMMs was further reduced, due to the incorporation of the highly 
$\mathrm{CO}_{2}$-soluble IL, while $\mathrm{CO}_{2}$ solubility remained constant, compared with the two-component ETS-10/CS MMM. This accounts for the $\mathrm{CO}_{2}$ affinity of the novel membrane materials synthesized in this work.

The permeation data obtained experimentally in this work are plotted against the Robeson's upper bound in Figure 5. There is a monotonous increase in permeability and selectivity, as the pure CS matrix is hybridized by the introduction of the small amounts of IL, ETS-10, and both. The highest permeability of the ETS-10/IL/CS MMMs is due to the IL/CS continuous phase and interaction of the IL both with particles and polymer [11]. The loading amount is kept constant as low as 5 wt \% with respect to CS concentration, and there is no decrease in selectivity observed, the MMM performance almost reaching that of other membrane morphologies, such as IL supported membranes (SILM) and purely inorganic ETS-10 tubular membranes.

Table 4. Solubility $(S)$ of measured gases in MMMs.

\begin{tabular}{|c|c|c|c|}
\hline $\begin{array}{c}\text { Membrane } \\
\text { material }\end{array}$ & $\begin{array}{c}S\left(\mathrm{CO}_{2}\right), \\
\mathrm{cm}^{3}(\mathrm{STP}) / \mathrm{cm}^{3} \mathrm{cmHg}\end{array}$ & $\begin{array}{c}S\left(\mathrm{~N}_{2}\right), \\
\mathrm{cm}^{3}(\mathrm{STP}) / \mathrm{cm}^{3} \mathrm{cmHg}\end{array}$ & $\begin{array}{c}\text { Solubility Selectivity } \\
\left(\mathrm{CO}_{2} / \mathbf{N}_{2}\right)\end{array}$ \\
\hline $\mathrm{IL}$ & 0.0114 & $-{ }^{a}$ & $-{ }^{\mathrm{a}}$ \\
\hline ETS-10 & 0.1019 & $-{ }^{a}$ & $-{ }^{a}$ \\
\hline $\mathrm{CS}$ & 0.0767 & 0.0050 & 15.34 \\
\hline $\mathrm{IL} / \mathrm{CS}$ & 0.5065 & 0.0255 & 19.84 \\
\hline ETS-10/CS & 0.0798 & 0.0029 & 27.58 \\
\hline ETS-10/IL/CS & 0.0724 & 0.0019 & 38.48 \\
\hline
\end{tabular}

Figure 5. Comparison of $\mathrm{CO}_{2}$ permeability and $\mathrm{CO}_{2} / \mathrm{N}_{2}$ selectivity for the CS-based MMMs with Robeson's upper bound [8A]. Void symbols represent the membranes where $\mathrm{CS}$ is the continuous phase, filled symbols those where the continuous phase is constituted by IL/CS. Dense CS, IL, and ETS-10 are literature pair values for dense CS membranes [38], supported [emim][Ac] membranes on a porous PVDF disk [22] and pure ETS-10 inorganic membranes on porous alumina supports [21], respectively.

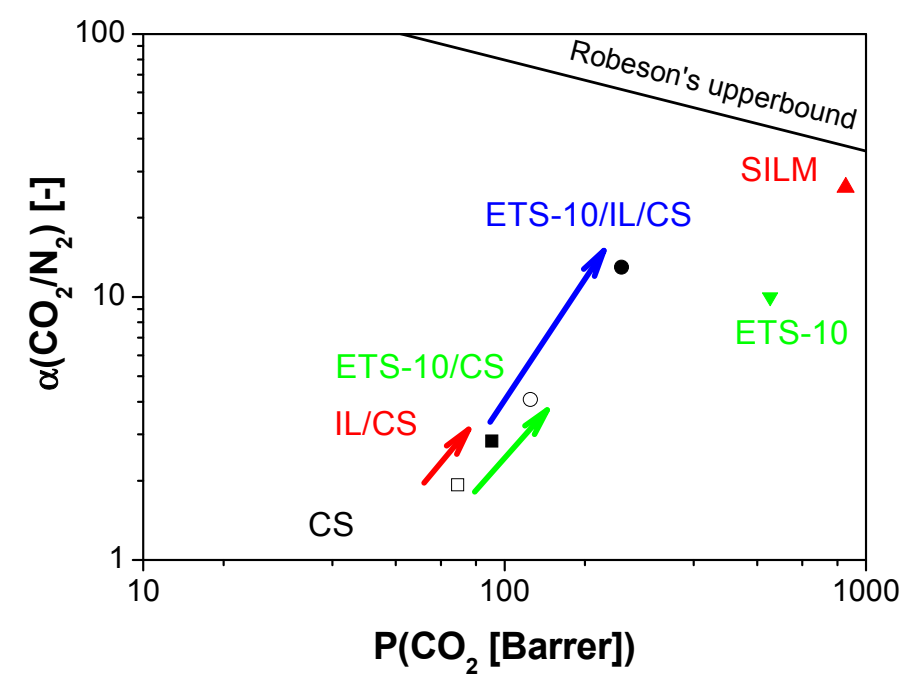

Hence, the addition of IL in just a small amount in the membrane not only increased permeability, but also seemed to act as intermediate medium imparting improved adhesion between the CS continuous polymer and the ETS-10 dispersed nanoparticles as well as enhanced $\mathrm{CO}_{2}$ affinity. 


\section{Experimental Section}

CS (coarse ground flakes and powder, Sigma-Aldrich) with a deacetylation degree higher than 75 wt $\%$ and high viscosity in 1 wt \% acetic acid/water was used as purchased. This CS product provides high density of amino groups for $\mathrm{CO}_{2}$ separation [38].

The ETS-10 nano-crystals were prepared according to a hydrothermal synthesis method previously reported [16,39] using $\mathrm{TiO}_{2}$-anatase (powder, $99.8 \mathrm{wt} \%$, Aldrich, Madrid, Spain) and sodium silicate solution (27 wt \% $\mathrm{SiO}_{2}, 8 \mathrm{wt} \% \mathrm{Na}_{2} \mathrm{O}$, Merck, Barcelona, Spain) as $\mathrm{Ti}$ and $\mathrm{Si}$ source, respectively. In a typical synthesis, $35.06 \mathrm{~g}$ of parent gel with molar composition $5.6 \mathrm{SiO}_{2}: 1 \mathrm{TiO}_{2}: 4.6 \mathrm{Na}_{2} \mathrm{O}: 1.9 \mathrm{~K}_{2} \mathrm{O}: 137$ $\mathrm{H}_{2} \mathrm{O}$ were poured into a Teflon-lined autoclave and submitted to hydrothermal synthesis at $230{ }^{\circ} \mathrm{C}$ for $24 \mathrm{~h}$. The autoclave was then removed from the oven and quenched under cold tap water to room temperature. The solid was washed and centrifuged at least 3 times, and dried at $100{ }^{\circ} \mathrm{C}$ overnight to recover about $2.8 \mathrm{~g}$ of final product. This product has a particle size of $a=b=0.32 \pm 0.06 \mu \mathrm{m}$ and $c=0.41 \pm 0.22 \mu \mathrm{m}$ and a BET surface area of $253 \pm 7 \mathrm{~m}^{2} / \mathrm{g}[19]$.

\subsection{Membrane Preparation}

The procedure to prepare each type of CS MMM is as follows. First, CS $2 \mathrm{wt} \%$ solutions were first dissolved in $2 \mathrm{wt} \%$ acetic acid (glacial, Panreac) aqueous solutions under stirring at $80{ }^{\circ} \mathrm{C}$ for $24 \mathrm{~h}$ at reflux conditions. The CS solution obtained was filtered to remove insoluble impurities and degassed in an ultrasonic bath before $10 \mathrm{~mL}$ on a polystyrene Petri dish and evaporating at room temperature for 2-3 days. CS membranes were then removed from the Petri dish. A $15.55 \mathrm{~cm}^{2}$ membrane was cut from the film for gas permeation and neutralized in $1 \mathrm{M} \mathrm{NaOH}$ and rinsed with abundant distilled water and dried at $4{ }^{\circ} \mathrm{C}$ before $\mathrm{CO}_{2}$ and $\mathrm{N}_{2}$ permeation experiments in order to ion-exchange the $\mathrm{NH}_{3}{ }^{+}$functional groups of the polymer matrix. Excess water was carefully removed by blotting the membrane, for diluted $\mathrm{CO}_{2}: \mathrm{N}_{2}: \mathrm{H}_{2}$ mixtures simulating flue gas streams [14,15]. Its mechanical stability has nevertheless been tried to improve by coating on a porous polysulfone support [16], organic chemical crosslinking [17], and physical mixing with zeolite particles [18]. Facilitated transport in the solid matrix is expected to increase the stability as well, and MMM with $5 \mathrm{wt} \%$ ETS-10 loading were prepared as reported elsewhere [18]. ETS-10 particles were first dispersed in distilled water (proportion 1:100 wt/wt) in an ultrasound bath for $10 \mathrm{~min}$ at room temperature. Then, CS solution (10 $\mathrm{mL}$ ) was added and treated in ultrasound bath for $15 \mathrm{~min}$ until a homogenous white dispersion was obtained and cast as described above.

IL/CS membranes were prepared with a nominal $5 \mathrm{wt} \%$ IL loading with respect to CS. In a typical synthesis: $0.042 \mathrm{~g}$ of IL (97 wt \%, Sigma-Aldrich, Madrid, Spain) were added to the $10 \mathrm{~mL} \mathrm{CS}$ solution and stirred overnight before casting in a similar manner as the pure CS membranes.

For the three-component ETS-10/IL/CS MMMs, the preparation method was similar to that employed for ETS-10/CS MMMs, using the IL/CS mixture as continuous phase.

\subsection{Characterization Methods}

Thicknesses were measured using a Mitutoyo Digital Micrometer with an accuracy of $0.001 \mathrm{~mm}$ after removal from the Petri dishes, measured in five points covering the whole membrane surface, 
before $\mathrm{NaOH}$ neutralisation treatment. The thicknesses of selected membranes were measured before and after the permeation measurement in order to check the validity of these values and the structural stability of the membranes upon permeation.

The experimental density values of the membranes $\left(\rho_{\mathrm{m}}\right)$ were measured gravimetrically from the electronically measured weight of the circular film and the calculated volume.

Thermo gravimetric analyses (TGA) were performed in a DTG $60 \mathrm{H}$ Shimadzu instrument (Japan) in air from 25 to $700{ }^{\circ} \mathrm{C}$ at a heating rate of $10{ }^{\circ} \mathrm{C} / \mathrm{min}$, in order to study the thermal stability of the resulting membranes. The decomposition temperature was calculated as the temperature at which $5 \%$ weight loss occurs. The gas solubility of the membranes was evaluated by $\mathrm{CO}_{2}$ and $\mathrm{N}_{2}$ adsorption measured gravimetrically at the same thermo balance mentioned above, which is equipped with a FC60A flowmeter (Shimadzu, Japan). The sorption experiments were conducted isothermally at $25{ }^{\circ} \mathrm{C}$ for 3 and $4 \mathrm{~h}$, under $\mathrm{CO}_{2}$ and $\mathrm{N}_{2}$ flow, respectively and a gas pressure of 5 bar. DSC analyses were carried out in a DSC 822 apparatus from Mettler Toledo, belonging to the Universidad de Zaragoza (Zaragoza, Spain). The samples were heated at $10{ }^{\circ} \mathrm{C} / \mathrm{min}$ from 100 to $300{ }^{\circ} \mathrm{C}$, after $1 \mathrm{~min}$ at $100{ }^{\circ} \mathrm{C}$, twice, to elucidate the glass transition in the second run.

The mechanical resistance of the membranes was measured by the tensile strength and the elongation at break of 5-10 of $5 \mathrm{~mm}$ wide samples of the membrane materials in a Universal Testing Machine (Zwick/Roell, Ulm, Germany) with a head load up to $2.5 \mathrm{kN}$ and $5 \mathrm{~mm} / \mathrm{min}$.

IR spectra were recorded on a Perkin Elmer Spectrum 100 FTIR spectrometer (Concord, Canada) with a resolution of $4 \mathrm{~cm}^{-1}$ and 32 scans, at the Universidad de Málaga (Málaga, Spain). The solid samples were measured after dilution in $\mathrm{KBr}$ pellets. For the membrane samples, these were dried at $100{ }^{\circ} \mathrm{C}$ for at least $2 \mathrm{~h}$ and grinded for 5 min prior to pellet preparation. The IL sample was sandwiched drop wise into two thin pieces of glass and measured using an optical transmission cell. All windows used were planar and compatible with infrared wavelengths.

Gas permeation was carried out with pure $\mathrm{N}_{2}$ and $\mathrm{CO}_{2}$ at room temperature in a constant volume system. Membranes were placed in the permeation cell, and tested for $\mathrm{N}_{2}$ first and then $\mathrm{CO}_{2}$. An average of 10 experimental runs were performed for each membrane composition. The experimental runs were left no more than $3 \mathrm{~h}$ in order to keep the membrane at constant operation conditions (relative humidity, pressure and driving force), and only the results obtained for the membranes that were still flexible when removed from the module were taken into account. In a typical run, gas was fed to permeate and feed compartments, which took a few minutes. Then, gas feed was closed and the vent valve was opened to empty the permeate compartment and generate the driving force across the membrane. Initial pressure was measured by a pressure transducer (Omega, Manchester, UK), and the pressure difference was monitored along the experimental run by a differential pressure transducer (Omega, Manchester, UK). The steady-state permeability $\left(1\right.$ Barrer $=10^{-10} \mathrm{~cm}^{3}$ (STP) $\mathrm{cm} \mathrm{cm}^{-2} \mathrm{~s}^{-1}$ $\left.\mathrm{cmHg}^{-1}\right)$ was calculated from the steady-state flux. Ideal selectivity $\alpha\left(\mathrm{CO}_{2} / \mathrm{N}_{2}\right)$ is defined as the ratio of the permeability values of the two gases, the faster gas permeability $\left(\mathrm{CO}_{2}\right)$ divided by the slower gas permeability $\left(\mathrm{N}_{2}\right)$, thus constituting an intrinsic property to compare different membrane materials. 


\section{Conclusions}

Mixed matrix membranes (MMMs) were prepared by adding a highly absorbing non-toxic ionic liquid, 1-ethyl-3-methylimidazolium acetate ([emim][Ac], IL), and a microporous titanosilicate ETS-10 nanoparticles to the continuous matrix of biopolymer chitosan (CS), and neutralising in $\mathrm{NaOH}$ solution to prepare the materials for $\mathrm{CO}_{2}$ affinity. The membrane material characteristics and permselectivity performance of the two-component membranes was compared with the three-component membrane and the pure CS membrane. The $\mathrm{CO}_{2}$ solubility increased upon addition of the IL and ETS-10 adsorbent. The mechanical strength was enhanced by the addition of ETS-10 particles, while the IL imparted flexibility to the membrane. The porosity and free volume were estimated from measured and theoretical densities. The void fraction of the ETS-10/IL/CS MMM was the largest obtained in this work, attributed to the interaction between IL, the polymer, and the porous ETS-10 particles. FT-IR spectra revealed a good interaction between the components in the MMMs, and a means of measuring the crystallinity to account for the real permeation area of the membrane, which is usually the amorphous part. These parameters (pore size, particle size and shape, interfacial adhesion, free volume, crystallinity) were correlated with the gas transport performance. This was evaluated by $\mathrm{CO}_{2}$ and $\mathrm{N}_{2}$ single gas permeation experiments. The selectivity of the three-component ETS-10/IL/CS MMMs was six times higher than pure CS membrane, showing in general a positive trend towards the attractive region of Robeson's upper bound.

\section{Acknowledgments}

The authors gratefully acknowledge the financial support from the Spanish Ministry of Economy and Competitiveness (MINECO) and the CCEI-University of Cantabria for the Ramón y Cajal programme (RYC-2011-08550) and MINECO for the project CTQ2012-31229. Joaquín Coronas (Universidad de Zaragoza) is also gratefully acknowledged for the use of DSC equipment and fruitful discussions.

\section{Author Contributions}

The experimental and theoretical part of this work was conducted by Clara Casado-Coterillo, who also prepared the draft of the article. Ángel Irabien was the head of the Sosprocan/DePro research group at the Universidad de Cantabria were the work was carried out. María del Mar López-Guerrero obtained and interpreted the FTIR spectra of the membrane materials at the Universidad de Málaga. All authors reviewed the several drafts of the manuscript.

\section{Conflicts of Interest}

The authors declare no conflict of interest.

\section{References}

1. Favre, E. Carbon dioxide recovery from post-combustion processes: Can gas permeation membranes compete with absorption? J. Membr. Sci. 2007, 294, 50-59. 
2. Merkel, T.C.; Lin, H.; Wei, X.; Baker, R. Power plant post-combustion carbon dioxide capture: An opportunity for membranes. J. Membr. Sci. 2010, 359, 126-139.

3. Gascón, J.; Kapteijn, F.; Zornoza, B.; Sebastián, V.; Casado, C.; Coronas, J. Practical approach to zeolitic membranes and coatings: State of the art, opportunities, barriers and future perspectives. Chem. Mater. 2012, 24, 2829-2844.

4. Pera-Titus, M. Porous Inorganic Membranes for $\mathrm{CO}_{2}$ Capture: Present and Prospects. Chem. Rev. 2014, 114, 1413-1492.

5. Bernal, M.P.; Coronas, J.; Menendez, M.; Santamaría, J. On the effect of morphological features on the properties of MFI zeolite membranes. Micropor. Mesopor. Mater. 2003, 60, 99-110.

6. Jiang, L.Y.; Chung, T.S.; Kulprathipanja, S. Fabrication of mixed matrix hollow fibers with intimate polymer-zeolite interface for gas separation. AIChE J. 2006, 52, 2898-2908.

7. Hamad, F.; Khulbe, K.C.; Matsuura, T. Comparison of gas separation performance and morphology of homogeneous and composite PPO membranes. J. Membr. Sci. 2005, 256, 29-37.

8. Robeson, L.M. The upper bound revisited. J. Membr. Sci. 2008, 320, 390-400.

9. Freeman, B.D. Basis of permeability/selectivity trade-off relations in polymeric gas separation membranes. Macromolecules 1999, 32, 375-380.

10. Hudiono, Y.C.; Carlisle, T.K.; Bara, J.E.; Zhang, Y.; Gin, D.L.; Noble, R.D. A three-component mixed-matrix membrane with enhanced $\mathrm{CO}_{2}$ separation properties based on zeolites and ionic liquid materials. J. Membr. Sci. 2010, 350, 117-123.

11. Hao, L.; Li, P.; Yang, T.; Chung, T.-S. Room temperature ionic liquid/ZIF-8 mixed-matrix membranes for natural gas sweetening and post-combustion $\mathrm{CO}_{2}$ capture. J. Membr. Sci. 2013, 436, 221-231.

12. Liu, L.; Chakma, A.; Feng, X. Gas permeation through water-swollen hydrogel membranes. J. Membr. Sci. 2008, 310, 66-75.

13. Ito, A.; Sato, M.; Anma, T. Permeability of $\mathrm{CO}_{2}$ through chitosan membrane swollen by water vapor in feed gas. Angew. Makromol. Chem. 1997, 248, 85-94.

14. El-Azzami, L.A.; Grulke, E.A. Carbon dioxide separation from hydrogen and nitrogen by fixed facilitated transport in swollen chitosan membranes. J. Membr. Sci. 2008, 323, 225-234.

15. El-Azzami, L.A.; Grulke, E.A. Parametric study of CO fixed carrier facilitated transport through swollen chitosan membranes. Ind. Eng. Chem. Res. 2009, 48, 894-902.

16. Kai, T.; Kouketsu, T.; Duan, S.; Kazama, S.; Yamada, K. Development of commercial-sized dendrimer composite membrane modules for $\mathrm{CO}_{2}$ removal from flue gas. Sep. Purif. Technol. 2008, 63, 524-530.

17. Xiao, S.; Feng, X.; Huang, R.Y.M. Trimesoyl chloride crosslinked chitosan membranes for $\mathrm{CO}_{2} / \mathrm{N}_{2}$ separation and pervaporation dehydration of isopropanol. J. Membr. Sci. 2007, 306, 36-46.

18. Casado-Coterillo, C.; Andrés, F.; Téllez, C.; Coronas, J.; Irabien, A. Synthesis and characterization of ETS-10/chitosan nanocomposite materials for pervaporation. Sep. Sci. Technol. 2014, doi:10.1080/01496395.2014.908921.

19. Casado, C.; Amghouz, Z.; García, J.R.; Boulahya, K.; González-Calbet, J.M.; Téllez, C.; Coronas, J. Synthesis and characterization of microporous titanosilicate ETS-10 obtained with different Ti sources. Mater. Res. Bull. 2009, 44, 1225-1231. 
20. Tiscornia, I.; Irusta, S.; Prádanos, P.; Téllez, C.; Coronas, J.; Santamaría, J. Preparation and characterization of titanosilicate Ag-ETS-10 for propylene and propane adsorption. J. Phys. Chem. C 2007, 111, 4702-4709.

21. Tiscornia, I.; Kumakiri, I.; Bredesen, R.; Téllez, C.; Coronas, J. Microporous titanosilicate ETS-10 membrane for high pressure $\mathrm{CO}_{2}$ separation. Sep. Purif. Technol. 2010, 73, 8-12.

22. Santos, E.; Albo, J.; Irabien, A. Acetate based supported ionic liquid membranes (SILMs) for $\mathrm{CO}_{2}$ separation: Influence of the temperature. J. Membr. Sci. 2014, 452, 277-283.

23. Blath, J.; Deubler, N.; Hirth, T.; Schiestel, T. Chemisorption of carbon dioxide in imidazolium based ionic liquids with carboxylic anions. Chem. Eng. J. 2012, 181-182, 152-158.

24. Alvarez-Guerra, M.; Irabien, A. Design of ionic liquids: An ecotoxicity (Vibrio fischeri) discrimination approach. Green Chem. 2011, 13, 1507-1516.

25. Ding, Z.-D.; Chi, Z.; Gu, W.-X.; Gu, S.-M.; Liu, J.-H.; Wang, H.-J. Theoretical and experimental investigation on dissolution and regeneration of cellulose in ionic liquid. Carbohydr. Polym. 2012, 89, 7-16.

26. Xie, H.; Zhang, S.; Li, S. Chitin and chitosan dissolved in ionic liquids as reversible sorbents of $\mathrm{CO}_{2}$. Green Chem. 2006, 8, 630-633.

27. Mi, F.-L.; Shyu, S.-S.; Wu, Y.-B.; Lee, S.-T.; Shyong, J.-Y.; Huang, R.-N. Fabrication and characterization of a sponge-like asymmetric chitosan membrane as a wound dresssing. Biomaterials 2001, 22, 165-173.

28. Lee, S.B.; Ha, D.I.; Cho, S.K.; Kim, S.J.; Lee, Y.M. Temperature/pH-sensitive comb-type graft hydrogels composed of chitosan and poly(N-isopropylacrylamide). J. Appl. Polym. Sci. 2004, 92, 2612-2620.

29. Ji, Z.; Warzywoda, J., Jr. Competitive nucleation and growth in seeded batch crystallization of titanosilicate ETS-10 using Ti( $\left.\mathrm{SO}_{4}\right)_{2}$. Micropor. Mesopor. Mater. 2005, 81, 201-210.

30. Balau, L.; Lisa, G.; Popa, M.I.; Tura, V.; Melnig, V. Physico-chemical properties of chitosan films. Cent. Eur. J. Chem. 2004, 2, 638-647.

31. Zuo, G.; Wan, Y.; Wang, L.; Liu, C.; He, F.; Luo, H. Synthesis and characterization of laminated hydroxyapatite/chitosan nanocomposites. Mater. Lett. 2010, 64, 2126-2128.

32. Bernardo, P.; Jansen, J.C.; Bazzarelli, F.; Tasselli, F.; Fuoco, A. Gas transport properties of Pebax(R)/room temperature ionic liquid gel membranes. Sep. Purif. Technol. 2012, 97, 73-82.

33. Xu, D.; Loo, L.S.; Wang, K. Characterization and diffusion behavior of chitosan-POSS composite membranes. J. Appl. Polym. Sci. 2011, 122, 427-435.

34. Staroszczyk, H.; Sztuka, K.; Wolska, J.; Wojtasz-Pajak, A.; Kolodziejska, I. Interactions of fish gelatin and chitosan in uncrosslinked and crosslinked with EDC films: FTIR study. Spectrochim. Acta A 2014, 117, 707-712.

35. Shen, Y.; Lua, A.C. Preparation and characterization of mixed matrix membranes based on PVDF and three inorganic fillers (fumed nonporous silica, zeolite 4A and mesoporous MCM-41) for gas separation. Chem. Eng. J. 2012, 192, 201-210.

36. Jeazet, H.B.T.; Koschine, T.; Staudt, C.; Raetzke, K.; Janiak, C. Correlation of gas permeability in a metal-organic framework MIL-101(Cr)-polysulfone mixed-matrix membrane with free volume measurements by positron annihilation lifetime spectroscopy (PALS). Membranes 2013, 3, $331-353$. 
37. Rocha, J.; Anderson, M.W. Microporous titanosilicates and other novel mixed octahedral-tetrahedral framework oxides. Eur. J. Inorg. Chem. 2000, 2000, 801-818.

38. El-Azzami, L.A.; Grulke, E.A. Dual mode model for mixed gas permeation of $\mathrm{CO}_{2}, \mathrm{H}_{2}$, and $\mathrm{N}_{2}$ through a dry chitosan membrane. J. Polym. Sci. B 2007, 45, 2620-2631.

39. Mayoral, Á.; Coronas, J.; Casado, C.; Téllez, C.; Díaz, I. Atomic resolution analysis of microporous titanosilicate ETS-10 through aberration corrected STEM imaging. ChemCatChem 2013, 5, 2595-2598.

(C) 2014 by the authors; licensee MDPI, Basel, Switzerland. This article is an open access article distributed under the terms and conditions of the Creative Commons Attribution license (http://creativecommons.org/licenses/by/3.0/). 